\title{
Coronavirus 19 disease: A current medical emergency - Scientific emergency or scientific opportunism
}

\author{
Carlos A. Acosta-Olivo ${ }^{1 *}$ and José C. Jaime-Pérez ${ }^{2}$ \\ ${ }^{1}$ Traumatology and Orthopedics Service; ${ }^{2}$ Hematology Service. "Dr. José Eleuterio González" University Hospital, Universidad Autónoma de Nuevo \\ León, Monterrey, Nuevo Leon, Mexico
}

\section{What do we currently know about this disease?}

According to the World Health Organization, coronavirus includes a wide family of viruses that cause disease in animals and humans, causing from a common cold up to illnesses like severe acute respiratory syndrome (SARS). Coronavirus disease (COVID-19) is the most recent infectious disease caused by coronavirus, unknown up until the epidemic which began in Wuhan, China, in December 2019. The most common symptoms are fever, dry cough, and fatigue, while the less common include nasal congestion, headaches, conjunctivitis, diarrhea, loss of smell and taste, and rash or discoloration of the fingers or toes. Most people $(80 \%)$ recover without the need of hospital treatment ${ }^{1}$.

To date, as reported by the WHO, there are 4,628,903 confirmed cases, with 312,009 deaths, affecting 215 countries, areas o territories. Meanwhile, in Mexico, there are 47,144 reported cases with 5,045 confirmed deaths ${ }^{2}$.

Due to said medical urgency, it is necessary to have adequate and current scientific information, which allows us to understand and be able to treat it, allowing us to develop prevention measures and vaccines, thus limiting the exposure of vulnerable populations.

\section{What is the current panorama in the international scientific field?}

In journals unrelated in a direct way to the disease, there are delays in the reviews of the manuscripts, inevitably resulting in delays in their publication. This is understandable since the efforts conducted by physicians around the world are focused on stopping or limiting the extension of this disease. However, this does not necessarily occur with the journals that receive information regarding this disease, where the priority is given to those related to COVID-19, in the approval of randomized clinical protocols, as well as in the approval of systemic revisions and meta-analysis.

In this respect, fast track approvals can be authorized without the proper scientific evaluation.

To date, in May 2020, a large number of scientific articles related to the current pandemic of COVID-19 are reported in high-impact scientific journals. In the Journal of the American Medical Association (JAMA) magazine, there are 347 results; however, most results are opinions (122), and actual research comes in second place with 60 reports. Some of these researches only include a few patients, with a short follow-up period, a very short time frame between reception, acceptance, and online publication, i.e., in JAMA, there are publications

\section{Correspondence:}

${ }^{*}$ Carlos A. Acosta-Olivo

E-mail: dr.carlosacosta@me.com
Available online: $18-06-2020$

Date of reception: 04-05-2020

Date of acceptance: 07-05-2020 DOI: 10.24875/RMU.M20000042
Medicina Universitaria. 2020;22(2):46-47 www.medicinauniversitaria.org license (http://creativecommons.org/licenses/by-nc-nd/4.0/). 
of series of non-controlled cases with five patients for the treatment of those with severe symptoms, with preliminary results which showed clinical improvement (Shen et al., 2020) ${ }^{3}$.

What number of authors is usually considered adequate for the publication of a scientific article? Those who have participated actively in the conception, completion, and editing of a study, as well as its evaluation and authorization, are obvious and evident. Almost every journal requests this, and many of them even require a list of who participated in a study and their contributions, following the same study mentioned above, are the participation of 27 authors for a short follow-up of around 30-40 days in five patients adequate? We must avoid what is referred to as "scientific opportunism," in this special situation which we are going through, which is unprecedented in current times, times of scientific awareness, electronic times, and much greater internet distribution.

Registered to date in the web page clinicaltrials.gov, searching under the terms COVID-19 or SARS-Cov-2, there are 819 studies, of which 504 an intervention is performed, 301 are observational. There are 394 active protocols in the recruitment phase, while 19 have been completed. This tells us that in the next few months, we will be exposed to a large number of information concerning this disease, as the aforementioned protocols go through scientific filters.

And the publication time frame of the manuscripts? What is the amount of time considered convenient for the publication of a manuscript? This results from different factors, among which we have accessibility to manuscript reviewers, the number of editors and the amount of revisions conducted on the manuscripts, time of publication of the journals (weekly, monthly, bimonthly, and trimestral), and number of manuscripts available for a determined number of the journal, among others. All this would be considered a normal editorial process.
Publication time frames of the different journals for articles related to this infection have been reduced drastically, going from several months, even years, to weeks or days. This results in a probable decrease in scientific quality and revisions on this fast track; hence, a lack of scientific rigor. Apparently, we are filling ourselves with information on this disease; a lot of it is valid and of high quality, others are irrelevant.

That said, as a result of the amount of information we have and are currently obtaining, we may overlook publications with true scientific value. Seemingly and only in relation to this disease, COVID-19 publishing in high-impact scientific journals (New England Journal of Medicine, JAMA) would not be linked to the quality of the work, but with the scientific opportunism of the authors and/or editors to have current data about said disease. For this reason, we ought to be very attentive to published information, without losing the scientific rigor that makes us realize when a publication could help in our development. Let us avoid becoming opportunists or alarmists to say: i read it first... I heard someone said.

Our patients trust us and trust in our experience, in our scientific knowledge incorporated with our efforts and patience throughout the years. Let's avoid becoming a bad source of information; once we lose objectivity and quality in our profession, we lose everything.

\section{References}

1. World Health Organization. $Q$ and A on Coronaviruses (COVID-19). Geneva: World Health Organization. Available from: https://www.who.int/ emergencies/diseases/novel-coronavirus-2019/question-and- answers -hub/q-a-detail/q-a-coronaviruses. [Last accessed on 2020 May 04].

2. World Health Organization. Coronavirus Disease (COVID-19) Pandemic. Geneva: World Health Organization. Available from: https://www.who.int/ emergencies/diseases/novel-coronavirus-2019. [Last accessed on 2020 May 18].

3. Shen C, Wang Z, Zhao F, Yang Y, Li J, Yuan J, et al. Treatment of 5 critically ill patients with COVID-19 with convalescent plasma. JAMA. 2020;323(16):1582-9. 